\title{
The Participatory Design of a Sound and Image Enhanced Daily Planner for People with Aphasia
}

\author{
Karyn Moffatt ${ }^{\dagger}$, Joanna McGrenere ${ }^{\dagger}$, Barbara Purves ${ }^{\ddagger}$, and Maria Klawe $\mathrm{e}^{ \pm}$ \\ Department of Computer Science ${ }^{\dagger}$ \\ School of Audiology and Speech Sciences \\ University of British Columbia, Vancouver, BC, Canada \\ Engineering and Applied Sciences ${ }^{ \pm}$ \\ Princeton University, Princeton, NJ, USA \\ aphasia-ubc@cs.ubc.ca
}

aphasia-ubc@cs.ubc.ca

\begin{abstract}
Aphasia is a cognitive disorder that impairs speech and language. From interviews with aphasic individuals, their caregivers, and speech-language pathologists, the need was identified for a daily planner that allows aphasic users to independently manage their appointments. We used a participatory design approach to develop ESI Planner (the Enhanced with Sound and Images Planner) for use on a PDA and subsequently evaluated it in a lab study. This methodology was used in order to achieve both usable and adoptable technology. In addition to describing our experience in designing ESI Planner, two main contributions are provided: general guidelines for working with special populations in the development of technology, and design guidelines for accessible handheld technology.
\end{abstract}

Categories \& Subject Descriptors: K.4.2 [Computers and Society]: Social Issues - Assistive technologies for persons with disabilities; H.5.2 [Information Interfaces and Presentation]: User Interfaces - Evaluation/methodology, graphical user interfaces, prototyping, user-centered design

General Terms: Human factors, experimentation, design.

Keywords: Assistive technology, universal usability, multi-modal interaction, participatory design, handheld devices, cognitive disabilities.

\section{INTRODUCTION}

Aphasia is a cognitive disorder that affects about 1 million people in the United States [2] and 100,000 people in Canada [1]. Aphasia is usually acquired as a result of stroke, brain tumor, or other brain injury, and results in an impairment of language, that is, to the production and/or comprehension of speech and/or written language. Rehabilitation can reduce the level of impairment, but a significant number of individuals are left with a life-long chronic disability that influences a wide range of activities and prevents full reengagement in life.

Permission to make digital or hard copies of all or part of this work for personal or classroom use is granted without fee provided that copies are not made or distributed for profit or commercial advantage and that copies bear this notice and the full citation on the first page. To copy otherwise, or republish, to post on servers or to redistribute to lists, requires prior specific permission and/or a fee.

CHI 2004, April 24-29, 2004, Vienna, Austria.

Copyright 2004 ACM 1-58113-702-8/04/0004...\$5.00.
There is great variability of language abilities and impairments across individuals with aphasia. This variability results both from differences in severity and relative impairment of language modalities [6]. For example, some aphasic individuals have relatively good auditory and reading comprehension but very limited output in either speech or written language. Others may have fairly fluent speech, albeit with numerous semantic errors, accompanied by relatively poor comprehension of both spoken and written language. In addition, there can be accompanying deficits, depending on the site of lesion in the brain, including right visual field deficits and right hemiparesis and hemiplegia, which affect limb function [6].

By harnessing advances in computer technology and handheld devices, and building on the computer literacy of an ever increasing number of aphasic individuals, it seems feasible today to create assistive technologies that permit individuals with aphasia to re-engage in life, and to augment their autonomy and quality of life. A wide variety of assistive technologies are already available, mainly to facilitate therapeutic efforts and the recovery and maintenance of basic language functions, yet the number of reports of successful applications for people with aphasia remains quite limited. This observation contrasts with the successful harnessing of computer technology in the service of communication for non-aphasic, speech-impaired individuals, such as Stephen Hawking.

One reason for the lack of previous success may be that efforts have tended to focus on individuals with severe or profound aphasia, for whom efforts to develop effective alternative communication strategies have failed, rather than attempting to leverage the skills of those with some retained communicative ability [7]. Another reason that may have thwarted previous efforts is that they have focused on technologies that support basic communication functions rather than higher-level goals, that is, the specific communication activities for daily living that occur for aphasic individuals after hospital/therapy discharge. In a 1988 survey, $72 \%$ of individuals with aphasia who responded reported that they could not return to work, despite $50 \%$ of them having received over a year of speechlanguage therapy [2]. The long-term goal of the Aphasia Project is to fill this niche by creating and evolving highlevel applications that meet specific needs of aphasic individuals with residual communication abilities. 
The research documented in this paper investigates the design of a specific high-level computer application for people with aphasia. The application, identified through interviews with aphasic persons, is a multi-modal daily planner. The Enhanced with Sound and Images Planner, or ESI Planner, uses triplets of images, sound, and text to redundantly encode information for the user. The research was conducted in two phases: a participatory design phase where ESI Planner was iteratively developed with input from aphasic participants, and an evaluation phase where an experimental study was performed to assess the effectiveness of the resulting design. Several guidelines emerged from this work that are relevant to other researchers working with special populations.

\section{RELATED WORK}

Two general areas that are relevant to our work are the design of technology for people with aphasia, and the evaluation of assistive technology. Research in the development of technology for people with aphasia has focused predominantly on devices to assist in communicative exchanges. While this is an obvious need for people with aphasia, it is not the only way that technology can support their daily activities. Nonetheless, much can be learned from the work done on development of communication devices.

To date, essentially all assistive technology proposed for persons with aphasia has been in the form of augmentative and alternative communication devices (AAC devices). Aphasic individuals tend to retain the ability to recognize image-based representations of objects, so various devices have been developed that build on this remaining ability. In these systems, each word/concept has a tri-modal representation, consisting of an image form, a sound form, and a visual-letter form. The user is able to search through the image library to retrieve a desired item, and once selected, its letter and sound forms are made available for use in communicating with others. These types of AAC devices may assist aphasic individuals who have few, if any, other communication options to express basic needs. However, they do little to leverage the skills of aphasic individuals who have some communicative ability [3].

In recent years, several investigators have applied computer technology to meet specific needs of aphasic individuals with some retained communicative ability. While in some instances such work has been undertaken in the context of individualized rehabilitation programs [5], others have developed and tested systems across users. For example, a prototype e-mail system using four different interfaces was tested with several cognitively disabled users, including individuals with aphasia [10]. In contrast, the TalksBac system [12] and a related system, PROSE [13], were developed specifically for persons with aphasia, in order to leverage their abilities to participate in conversation. TalksBac guides users through a continually updated selection of short sentences and phrases that can be read aloud via a speech synthesizer during conversation. PROSE, designed to be used in conjunction with TalksBac, allows the aphasic user to introduce pre-recorded stories into conversations. Both systems rely heavily on the availability and willingness of familiar partners, or caregivers, to manage and update entries in the system on an ongoing basis.

TalksBac was evaluated by four aphasic individuals for nine months. It was found to be helpful for two of the four participants; this success was attributed to its ability to fill a need that had not already been filled by the development of effective alternative communication strategies [12]. PROSE was compared for one individual against two other strategies and found to be effective in augmenting her conversational participation [13]. These findings, though limited, suggest the potential of computer technology to address specific needs of people with aphasia.

Stevens and Edwards discuss the difficulties in working with special populations in their evaluation of Mathtalk [9]. They highlighted several challenges in evaluating assistive technology including the inappropriateness of controlled laboratory experiments for highly heterogeneous populations, the difficulty of acquiring a sufficient sample of the population, and the unavailability of appropriate control conditions. Each of these challenges, although especially the first two, has also been a challenge inherent in our work.

\section{PHASE I: PARTICIPATORY DESIGN}

Most people with aphasia have difficulty reading and writing. ESI Planner is a multi-modal daily planner designed to enable individuals with aphasia to independently manage their schedules. It incorporates triplets of images, sound, and text to represent appointment data. We hypothesized that these triplets would make it easier for people with aphasia to comprehend the information presented within a daily planner. This hypothesis was based on knowledge that people with aphasia generally retain their ability to recognize images [11], and anecdotal evidence from our participants suggesting that reading may be easier when the text is concurrently read aloud to them.

The first phase of the research used participatory design methodology, a process that uses early and continual participation of the intended users to produce a technology that will realize better acceptance and better suit the needs of its users. Figure 1 shows a timeline for this phase and highlights the iterative nature of this work. Although we did not follow a strictly sequential process, for the purposes of clarity we describe this phase in four steps: brainstorming, lowfidelity paper prototyping, medium-fidelity software prototyping, and high fidelity software prototyping.

Ideally, the participants in this phase of the research would have remained constant throughout the work. Unfortunately, our initial participant, $A B$, who had motivated the ESI planner, passed away from brain cancer before the completion of the preliminary design. Thus, three surrogate design members, SM, SS, and MP, were recruited to fill $\mathrm{AB}$ 's role and ensure continued progress. 
$\begin{array}{cc}\begin{array}{c}\text { Brainstorming and low-fi } \\ \text { prototyping with } A B\end{array} & \text { Low-fi prototyping of appointment brows } \\ \text { ing with } 4 \text { non-aphasic participants }\end{array}$

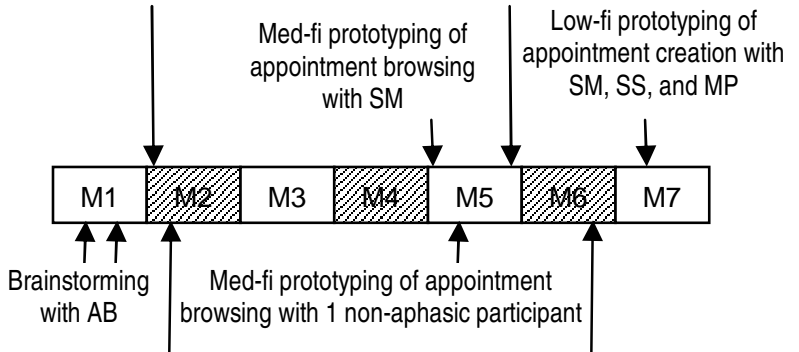

Brainstorming and informal evaluation of commercial day planner with SM

Med-fi prototyping of appointment browsing with SM, SS, and MP

Figure 1: Timeline for the participatory design phase in months (where month is denoted by $M x$ ).

Due to the large variability in impairments across people with aphasia, none of these individuals had exactly the same difficulties as $\mathrm{AB}$, although all felt that improvements could be made to text-only planners. In fact, one of the participants, who had initially felt he would not benefit from our design, became enthusiastic as the research progressed and he discovered the potential for an enhanced planner to aid him in communicating his daily activities to others.

\section{Brainstorming}

We began by brainstorming with one aphasic individual, $\mathrm{AB}$, to identify areas where technology could be used to support her daily activities. AB was a middle-aged professional woman who was highly computer literate. She was an active and energetic individual both before and after she acquired aphasia, and although she had difficulty finding words and was largely unable to read or write, she maintained relatively fluent speech. One of several needs identified was for a daily planner that would allow $\mathrm{AB}$ to manage her appointments. Prior to acquiring aphasia, AB had used a PDA-based calendar to manage her busy life. However, as AB's aphasia, which resulted from brain cancer, progressed $^{1}$ she was becoming increasingly reliant on a caregiver to manage her appointments. Because $\mathrm{AB}$ wished to maintain an independent lifestyle, it was essential that she be able to manage her planner without assistance.

AB's problem with traditional paper or electronic daily planners was twofold. First, the input of appointment data via writing, typing, or tapping was slow and difficult. Attempts often resulted in frustration and resignation. Second, the representation of appointment data as text made it difficult for her to recognize and interpret the information. This was true even for appointments she had entered herself, as her language skills were inconsistent and unreliable.

\footnotetext{
${ }^{1}$ In contrast, individuals with stroke related aphasia do not experience a further decline in their language skills after their stroke, with rehabilitative efforts typically leveling off around one year.
}

\section{Low-Fidelity Paper Prototyping}

Based on our brainstorming sessions with $\mathrm{AB}$, we created a series of initial low-fi paper prototypes to evaluate different design ideas (one example is shown in Figure 2a.). Standard low-fi prototype evaluation, which includes the think-aloud protocol, proved very difficult for $\mathrm{AB}$. While her speech remained relatively fluent, it often lacked sufficient detail for her to give a specific account of how she would interact with the proposed interfaces. Furthermore, she was very concerned with her physical ability to interact with the proposed device, an aspect of the design for which paper prototyping provides no insight.
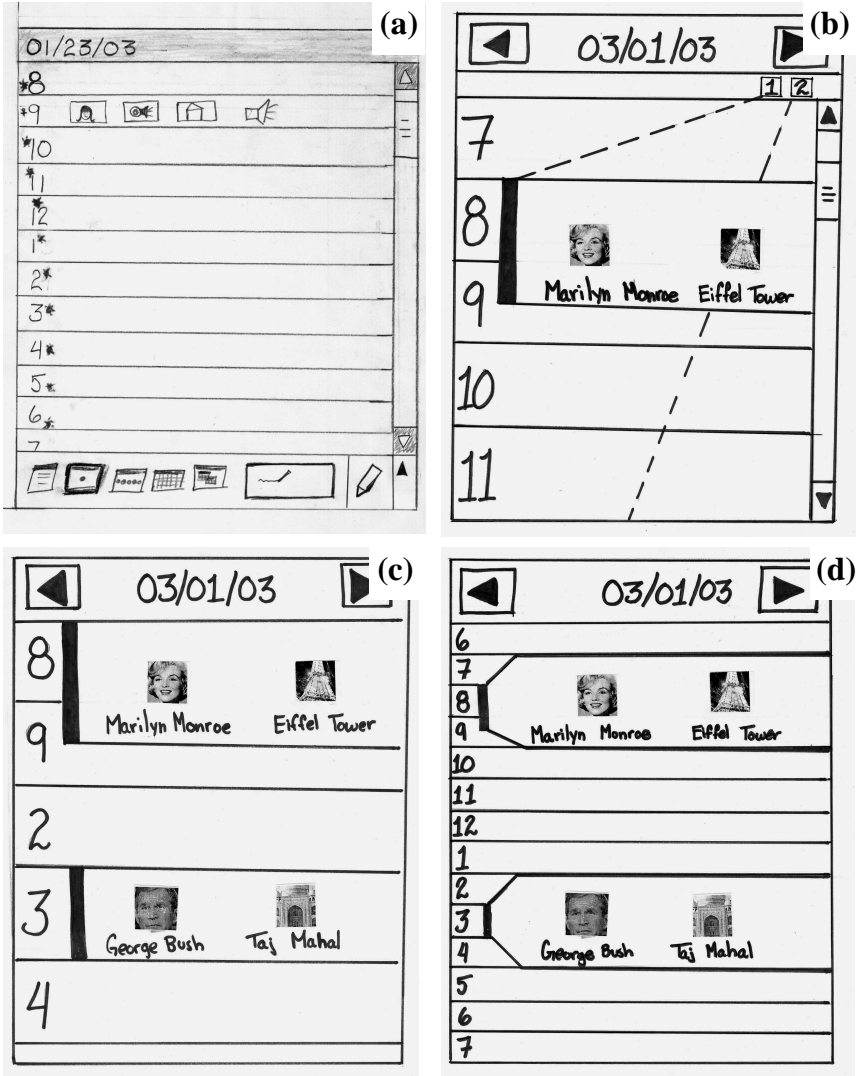

Figure 2: Paper prototypes: (a) initial prototype, (b) emphasized scroll, (c) dynamic timeline, \& (d) detail in context.

Paper prototypes were, however, useful for discussing specific aspects of the design relative to AB's abilities and for stimulating general design discussion. For instance, with respect to the prototype shown in Figure $2 \mathrm{a}, \mathrm{AB}$ commented that while text is difficult she would not have trouble with the numbers used to display time. She also noted that while the small suns used to highlight the time of day may be helpful, they would become a greater hindrance if they interfered with the numeric representation, suggesting a need for clear separation between images and text.

Despite its challenges, paper prototyping was used multiple times in our design process. As explained in the subsequent section, we found many design flaws in our first mediumfidelity prototype that did not appear to be specific to aphasia. After confirming our suspicions by testing our proto- 
type with one non-aphasic individual, we decided to step back to paper prototyping, this time with non-aphasic participants. We tested three different designs using four nonaphasic participants (Figures 2b-2d). Our rationale for taking this course of action was that by removing general design flaws we could better use our time with aphasic participants to focus on aphasia-specific aspects of the design.

\section{Medium-Fidelity Software Prototyping}

Our first prototype for ESI Planner had a similar design to Microsoft Pocket Outlook in that it assigned equal screen real estate to each hour of the day, which can require vertical scrolling, even to view appointments that fall within the 6 am to $8 \mathrm{pm}$ timeframe. However, in our first session, it became clear that for browsing tasks, the combination of searching through the days, and scrolling within a day to find appointments that are not initially visible was very difficult. Suspecting that this difficulty had nothing to do with aphasia, we ran the same tasks with one non-aphasic individual and found the same problems. At this point, as previously mentioned, we went back to paper prototyping using non-aphasic individuals to test three interfaces: an emphasized scroll version that clearly indicated when appointments were "hidden" (Figure 2b), a dynamic timeline version similar to that used in Palm planners that displayed only hours for which appointments existed and thereby minimized scrolling (Figure 2c), and a detail in context design (Figure 2d). The detail-in-context design proved to be the clearest design, and is therefore the one we used. As shown in Figure 3, the left hand side of the screen sets the context, highlighting which parts of the day are booked, while the right hand side gives the appointment details.

\section{High-Fidelity Software Prototyping}

The participatory design phase of this research resulted in a high-fidelity prototype of ESI Planner, which was subsequently evaluated in an exploratory study. Figure 3 shows an image of the final design. Each screen displays a single day from 6:00am to 8:00pm for which a maximum of 5 appointments can be scheduled. Although this aspect of the design could be seen as limiting, from our discussions with speech-language pathologists and our brainstorming sessions with participants, these choices seemed to meet the needs of our user population.

Design flaws were uncovered during the formal evaluation, although further studies are needed to determine whether they are aphasia specific. Many study participants experienced problems with bi-directional controls such as the up/down arrows used for place selection in Figure 3. Seemingly confused by the two options, these users would alternate between the buttons, never advancing to the target. Also, multiple participants demonstrated hesitance in exploring the interface. This obstacle could perhaps be overcome by clearly indicating the effect of an action. For example, adding the next day's date to the date forward button could indicate more clearly the effect of tapping that button.

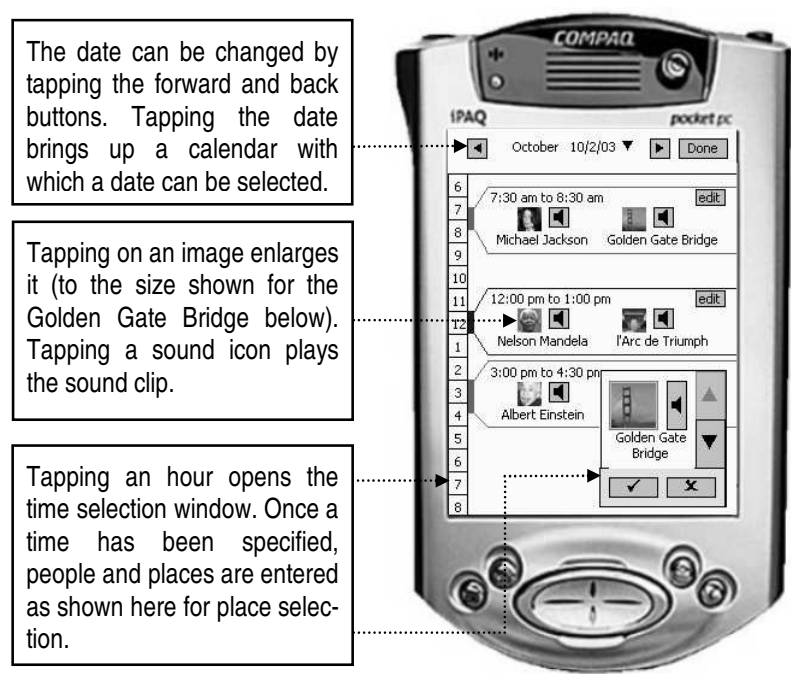

Figure 3: The ESI Planner interface showing appointments for October 2, 2003.

ESI Planner was implemented in embedded Visual Basic 3.0 using the Pocket PC 2002 SDK. It uses the Pocket Outlook Object Model as the backend for appointment data storage. All evaluations of ESI Planner were done using an HP iPAQ 5400 running Pocket PC 2002.

\section{PHASE II: EXPERIMENTAL EVALUATION}

An exploratory experiment was conducted to evaluate the ESI Planner interface relative to our goals of developing a usable high-level application that would better support the needs of aphasic users. In order to meet the challenges inherent in working with this population, this was not a traditional laboratory study. Some of the constraints of a traditional laboratory study, such as maintaining a consistent experimental environment, needed to be relaxed in order to accommodate the special needs of this population.

\section{Two Planner Conditions}

ESI Planner was compared with an equivalent text only electronic planner, NESI Planner (Not Enhanced with Sound and Images Planner). In this study, we wanted to specifically test our hypothesis that an interface using images and sound would better support aphasic individuals in appointment management tasks. Thus, the NESI planner interface retains as much of the ESI Planner interface as possible while removing sound and image functionality. Figure 4 shows the equivalent screen-capture of NESI Planner to that of the ESI Planner in Figure 3.

\section{Participants}

Nine aphasic individuals participated in the study. Our goal was to have eight participants complete the study, and one of the nine participants was unable to do so. Nonetheless, we believe his data provides valuable insights into the evaluation process, and thus we include it in our discussion. Participants ranged in age from 47 to 86 . They had a range of educational backgrounds from high school completion up to post-graduate education. There were 1 female and 


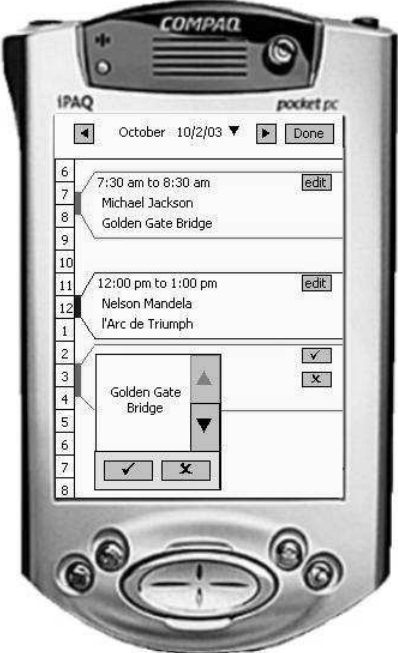

Figure 4: The NESI Planner interface showing the same data view as ESI Planner in Figure 3.

8 male participants. None of the 9 participants were part of the participatory design phase of the ESI Planner.

Participants were selected to be at least one year post onset to ensure a minimum level of stability had been reached in health and rehabilitation. Most had some experience with computers; only one had not used a computer previously. All possessed an interest and willingness to learn. While nine participants would be considered small in a traditional user study, it is a sizeable number for this population. The study was conducted in a location that was convenient to each participant, most often at a stroke or aphasia club to which they belonged. Two individuals, however, preferred to come to the university. While working with the planners, only the researchers and the participant were present. The ESI Planner was designed to be used independently, and therefore caregivers were not involved in the evaluation.

\section{Methodology}

Given the extensive individual differences inherent in our population, a within subjects design was chosen. We counterbalanced the order in which participants viewed the interfaces to control for learning effects. Based on recommendations from speech-language pathologists, we included two sessions, neither of which lasted more than 90 minutes. The first session, conducted by a computer science researcher, was the planner evaluation session; participants performed a set of tasks with one planner, took a break, and then completed an isomorphic set of tasks with the second planner. The second session comprised a speech and language assessment conducted by a certified speech-language pathologist.

A 90 minute evaluation session allowed participants to spend at most 30 minutes with each interface, with the remaining 30 minutes for interviews, and administrative details. The study design was piloted with SS from the participatory design phase, revealing that at most 10 tasks could be completed in a 30 minute trial.
One challenge was determining appropriate task scenarios. It would not have been realistic to test participants on the management of appointments with people and places they had never seen before, nor was it practical to create fully customized databases for each participant. We chose a compromise and constructed databases of 15 famous people and 15 famous places. At the start of the session, participants were given the opportunity to go through the databases and eliminate up to 5 unfamiliar faces and 5 unfamiliar places (No participant selected the maximum 5 unfamiliar entries in either category).

The 10 tasks were broken into three primary categories: retrieval, creation, and modification. In addition there was one compound task where the participant was asked to count the number of appointments matching a specific criterion (e.g., appointments with Marilyn Monroe) over a period of time. For each of the three categories, the participant was first given a demonstration of the task by the researcher and then given three similar tasks to perform. The first two of these tasks were presented in written form, but read aloud if necessary. The second task was considered to be a more reliable indication of the participant's ability to complete the task, as any misunderstandings could be clarified during the first task. The third task was given verbally with written cues if necessary and was designed to evaluate the participant's ability to manage the planner with auditory instructions only. Given the individual differences in participants' language abilities, these different task presentations were used in order to evaluate the effect of task presentation on participant response. Figure 5 shows an example appointment creation task. Pictures were used where possible to highlight information, and information was chunked so as to facilitate reading comprehension.

\begin{tabular}{|c|c|c|c|}
\hline \multicolumn{3}{|c|}{ Create an appointment } & $4 \mathrm{~A}$ \\
\hline With & Person: & \multicolumn{2}{|l|}{ Marilyn Monroe } \\
\hline At & Place: & \multicolumn{2}{|l|}{ Eiffel Tower } \\
\hline \multicolumn{4}{|c|}{ When? } \\
\hline & Date: & \multicolumn{2}{|c|}{ September 14, 2003} \\
\hline & Start Time: & \multicolumn{2}{|l|}{ 4:00pm } \\
\hline & End Time: & \multicolumn{2}{|l|}{$5: 15 \mathrm{pm}$} \\
\hline
\end{tabular}

Figure 5: Example of a written task used in the evaluation of ESI Planner.

Designing tasks such that the time for the participant to communicate the result would not dominate the task time was particularly challenging. For example, to test appointment retrieval the participant might be asked to find out with whom a particular appointment is scheduled. The desired measure in such a task is the time it takes the participant to determine with whom the appointment is scheduled, and thus should not include the time taken to communicate 
the result, which could vary significantly among participants and even among tasks for any one participant. Therefore, to assess task success we relied on self-assessment. Participants were instructed to take time before beginning each task to ensure that they understood the task. When they felt they understood, they were to begin by tapping a start button. This button switched the display to the planner interface and started the task timer. A done button was added to the interface for our study as shown in the upper right hand corner of the screen in Figures 3 and 4. When participants felt they had completed the task, they were to tap the done button. This stopped the timer, and hid the planner interface between tasks.

The 10 tasks were given to the participant one at a time. When 30 minutes had expired, the researcher indicated to the participant that it was time to stop and move on to the next part of the study.

Video recordings were used to capture interactions between the participant and the system, including unsuccessful screen taps that could not be captured in a system event log, and verbal interactions between the participant and the researcher. A listing of all commands issued and task times was recorded in a time-stamped file.

After both interfaces had been evaluated, the researcher conducted a structured interview to capture information which included participants' computer experience, daily planner usage, and interface preferences.

\section{Dependent Measures}

The following quantitative measures of performance were used for each of the two planners:

- Task time: sum of all task times

- Tasks correct: number of tasks completed correctly

- Tasks incorrect: number of tasks completed incorrectly

The qualitative self-reported measures captured in the interview and used for ranking the planners were as follows:

- Fastest: which planner was fastest

- Easiest: which planner was easiest to use

- Preferred: which planner was preferred overall

- Long term: which planner would be preferred if the participant had a longer time to spend using it

\section{Individual Differences}

Participants' language abilities were assessed using the Western Aphasia Battery [8], a standardized battery that is widely used to assess language impairments in aphasia. On the basis of assessment results, participants' abilities in the areas of speech, auditory comprehension, reading, and writing are described in terms of severity (see top of Table 1).

\section{Results}

On average, participants spent 17 minutes and 12 seconds doing tasks with ESI Planner, compared to 15 minutes and 47 seconds with NESI Planner (These figures include participants who reached the 30 minute time limit, however, the same number of participants reached the limit in each condition - 3 ESI, 3 NESI). While the task time comparison is not statistically significant, it may suggest that ESI Planner takes longer to learn.

Participants did, however, complete significantly more tasks correctly with ESI Planner $(F(1,7)=10.3, p<.02)$. On average they correctly completed 7.9 tasks with ESI Planner, and only 6.8 tasks in the NESI Planner. So although ESI Planner may not be faster than NESI Planner, it does result in more accurate performance. Figure 6 shows a chart of the individual scores for tasks correct. In line with the finding that participants completed more tasks correctly with ESI Planner than with NESI Planner, participants completed fewer tasks incorrectly with ESI Planner, completing on average 0.9 and 1.1 tasks incorrectly with ESI Planner and NESI Planner respectively. However, this result is not statistically significant.

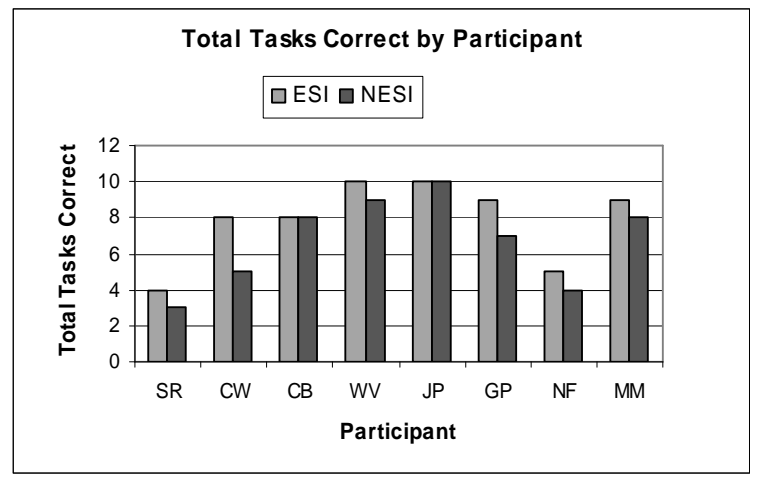

Figure 6: Number of tasks completed correctly in each interface by participant. $(N=8)$

We further analyzed the results to see if the format in which a task was presented (written vs. auditory) had an effect on task success. For this comparison, we considered the three auditory tasks given, and the second three of the six corresponding written tasks. However, as each of our participants was equally impaired in reading and auditory comprehension no conclusions could be drawn from this data. Our belief is that presentation format needs to be customized to ensure that each participant's strengths are leveraged. However, further studies with participants specifically chosen to differ in reading and auditory comprehension would be needed in order to verify this.

Qualitatively, participants were asked to rank the planners in four categories: fastest, easiest, preferred, and long term. The results of these questions are shown in the bottom of Table 1. They are shown with the results of the language assessments as the combination of the two measures reveal some interesting results.

In general, participants were evenly divided in their preferences. However, when language assessments are taken into account, trends emerge that suggest a higher preference for ESI Planner for certain types of users. The 3 participants who consistently ranked NESI Planner higher had mostly mild deficits (WV, JP, GP). Of the 5 participants who 
Table 1: Top- speech and language classifications reflecting WAB scores as follows: mild (8-10), moderate (4-7), and severe (0-3). Bottom - self reported planner preferences. $(N=9)$

\begin{tabular}{l|ccccccccc}
\multicolumn{1}{c|}{ Measure } & SR & CW & CB & WV & JP & GP & NF & MM & ET* \\
\hline Speech & mild & severe & moderate & mild & mild & mild & severe & mild & severe \\
Auditory & moderate & moderate & mild & mild & mild & mild & moderate & mild & severe \\
Reading & moderate & moderate & mild & mild & mild & mild & moderate & mild & moderate \\
Writing & severe & severe & severe & mild & moderate & mild & severe & mild & moderate \\
\hline Fastest & Neither & ESI & ESI & NESI & NESI & NESI & ESI & ESI & n/a \\
Easiest & Neither & ESI & ESI & NESI & NESI & NESI & NESI & ESI & n/a \\
Preferred & ESI & ESI & ESI & NESI & NESI & NESI & ESI & ESI & n/a \\
Long Term & ESI & ESI & NESI & NESI & NESI & NESI & ESI & ESI & n/a
\end{tabular}

*As ET did not complete the evaluation session his data is provided here for observational purposes only

preferred ESI Planner, 4 had two or more moderate or severe classifications (SR, CW, CB, NF). Although we cannot conclusively say anything about the influence of any one of the language ratings, we believe reading is most likely the predominate factor in influencing this split. For participants with only mild reading deficits, navigating NESI Planner was relatively easy; however, when reading is at least moderately impaired ${ }^{2}$, the image and sound support ESI Planner provides became important for task success.

A final observation refers to participant ET, who was unable to complete the study. ET had a severe auditory comprehension deficit, whereas all other participants had only a mild or moderate deficit in this category. This, combined with his severe or moderate deficits in all other areas, may have made it difficult for ET to communicate with the researcher and to understand the tasks presented. Based on a conversation with ET's caregiver after the session, we strongly suspect that his difficulties with the experimental evaluation do not reflect his actual ability to learn or use the interfaces presented. Rather, we believe ET required more time to acclimatize himself to the device and the tasks, and more support from the researchers. This finding highlights the limitations of experimental evaluation and reinforces the need for alternative evaluation techniques.

The results presented here reflect a preliminary analysis of the data. Ongoing analyses of the video data and log files are under way.

\section{IMPLICATIONS}

The implications of this work have been divided into two broad categories: guidelines for working with special populations, and guidelines for designing accessible handheld technology. Each of these topics is discussed in turn.

\footnotetext{
2 There were no participants in this study with severe reading impairments; we predict that for such individuals, preferences would be the same or stronger as for those with moderate reading impairments
}

\section{Guidelines for working with Special Populations}

Working with special populations presents many challenges. Within our work with aphasic persons, the most notable challenges included interpreting data from a population with large individual differences, recruiting sufficient participants, addressing mobility and transportation issues, and communicating with participants. The guidelines below relate to methodological insights that emerged throughout our work and that address these challenges.

\section{Assess abilities through standardized tests}

Speech and language assessments proved invaluable. They provided insights into the results that would not otherwise have been apparent. It is important to note that these assessments provided more than mere confirmation of the researchers' informal intuition. In many situations, the researchers were surprised by the results of the assessment; many aphasic individuals have developed sufficient compensatory skills to mask the extent of their deficits.

\section{Connect with existing groups and organizations}

The cooperation and assistance of aphasia and stroke clubs aided immeasurably in the execution of this research. They facilitated recruitment by helping us contact participants and mitigated transportation needs by allowing us to use their facilities as a common place where we could meet with several participants in one visit. It is important to note, however, that this was not a perfect solution. Performing the research off-site meant giving up most of the benefits of a controlled laboratory. Aphasia centers and stroke clubs do not generally have extra resources, and thus space offered to us was typically the personal office of one of the organizers. While the club facilitators were sensitive to the needs of the researchers, they also had their own jobs to perform. This ultimately led to several unavoidable disturbances throughout the course of the study.

\section{Gain practical experience with the target population}

The most difficult challenge in this work was communicating effectively with participants. Extra time had to be allot- 
ted to ensure participants had sufficient time to fully understand the tasks and ask questions. While this was a challenge throughout all phases of this research, it was particularly significant during the experimental evaluation phase, where timing was critical. In that stage, the sensitivity developed by the researchers during the participatory design phase was critical to minimizing the effect of communication barriers on the research outcome.

\section{Guidelines for Accessible Handheld Technology}

Modifications to increase the accessibility of the keyboard date back to the 1980's [4]. However, similar accessibility options are not available for handheld devices. The tap interaction of the handheld is problematic for many users with motor control impairments ${ }^{3}$ as it provides no support for targeting. With a mouse, one can carefully line up the pointer until it overlaps with the desired target, and then push the mouse button, but with tapping this is not possible. Some of our participants managed to work around this problem by using inactive space around buttons as a "landing zone" from which they could drag the stylus to the target. Thus it is important to ensure that accessible stylusbased technology is designed with sufficient inactive space available to permit targeting.

Another problem identified with the handheld device was tap sensitivity. Motor control limitations caused some individuals to tap repeatedly, causing unexpected behavior in the system. Unlike on the desktop, where accessibility options allow users to customize the sensitivity of key input, there is no such functionality on the Pocket PC operating system. Such functionality is required if these systems are to be used for assistive technologies.

\section{CONCLUSIONS AND FUTURE WORK}

We have presented a description of the process used to design an accessible multi-modal daily planner for people with aphasia. Participatory design followed by a formal evaluation was central to ensuring that the resulting technology was usable by the target population. The exploratory evaluation revealed that the multi-modal planner did improve the ability of aphasic participants to manage appointment data and was preferred by participants who had moderate impairments.

It is not uncommon for assistive technologies to fail to be adopted even after demonstrating success in clinical or laboratory settings [3]. The participatory process used to design the ESI Planner, together with our lab-style evaluation, give us confidence that we are on the right path to achieving an adoptable technology; however, more evaluation is required. Our next steps will be to refine the design based on findings from the current study and then work towards a field evaluation. Given the wide range of abilities

\footnotetext{
${ }^{3}$ While not strictly associated with aphasia, motor control impairments are common in the elderly population.
}

of our target population, we expect that deep customizability will be required in the final design.

\section{ACKNOWLEDGMENTS}

We thank the members and organizers of the BC Aphasia Centre, the Victoria Leap Program, and the Shaughnessy Stroke Club for their assistance in this research.

\section{REFERENCES}

[1] The aphasia institute: What is aphasia? Available 09/2003 at <http://www.aphasia.ca/about/whatis.html>

[2] Impact of aphasia on patients and family. Available 09/2003 at <http://www.aphasia.org/NAAimpact.html>

[3] Beukelman, D.R., \& Mirenda, P. (1998). Augmentative and alternative communication: Management of severe communication disorders in children and adults $\left(2^{\text {nd }}\right.$ Ed.). Paul H. Brooks Publishing Co, Baltimore, MD.

[4] Buxton, W., Foulds, R., Rosen, M., Scadden, L., \& Shein, F. (1986). Human interface design and the handicapped user. Proceedings of CHI 1986, ACM Press, 291-297.

[5] Cole, E. (1999). Cognitive prosthetics: an overview to a method of treatment. NeuroRehabilitation, 12(1), 39-51.

[6] Goodglass, H., Kaplan, E. \& Barresi, B. (2001). The assessment of aphasia and related disorders ( ${ }^{\text {rd }} \mathrm{Ed}$.). Lippincott Williams, \& Wilkins, Philadelphia, PA.

[7] Hux, K., Manasse, N., Weiss, A., \& Beukelman, D. (2001). Augmentative and alternative communication for persons with aphasia. In Language Intervention Strategies in Adult Aphasia (4 ${ }^{\text {th }}$ Ed.) Lippincott Williams \& Wilkins, Philadelphia, PA, 675-689.

[8] Kertesz, A. (1982). Western Aphasia Battery. Grune and Stratton, New York, NY.

[9] Stevens, R.D., and Edwards, A.D.N. (1996). An approach to the evaluation of assistive technology. Proceedings of ASSETS 1996, ACM Press, 64-71.

[10] Sutcliffe, A., Fickas, S., Sohlberg, M. M., \& Ehlhardt, L. A. (2003). Investigating the usability of assistive user interfaces. Interacting with Computers, 15, 577602.

[11] Thorburn, L., Newhoff, M. \& Rubin, S. (1995). Ability of subjects with aphasia to visually analyze written language, pantomime, and iconographic symbols. American Journal of Speech-Language Pathology, 4, 174179.

[12] Waller, A, Dennis, F. Brodie, J., \& Cairns, A. Y. (1998). Evaluating the use of TalksBac, a predictive communication device for nonfluent adults with aphasia. International Journal of Language and Communication Disorders 33(1), 45-7.

[13] Waller, A. \& Newell, A. (1997). Towards a narrativebased augmentative communication system. European Journal of Disorders of Communication, 32, 289-306. 